\title{
Liver test abnormalities in patients with HIV mono-infection: assessment with simple noninvasive fibrosis markers
}

\author{
Rosa Lombardia, Robert Leverb, Colette Smith', Neal Marshall'b, Alison Rodgerc, Sanjay Bhaganib, \\ Emmanuel Tsochatzis ${ }^{\mathrm{a}}$
}

Royal Free Hospital and UCL, London, UK

\section{Abstract}

\section{Introduction}

In the era of effective antiretroviral therapy (ART), chronic liver disease is an important cause of morbidity and mortality

${ }^{a}$ UCL Institute for Liver and Digestive Health (Rosa Lombardi, Emmanuel Tsochatzis); ${ }^{b}$ Department of Infectious Diseases/HIV Medicine (Robert Lever, Neal Marshall, Sanjay Bhagani); 'CCL Research Department of Infection and Population Health (Colette Smith, Alison Rodger), Royal Free Hospital and UCL, London, United Kingdom

Conflict of Interest: None

Correspondence to: Emmanuel A. Tsochatzis, MD, PhD, UCL Institute for Liver and Digestive Health, Royal Free Hospital and UCL, Tel.: +44 2077940 500, e-mail: e.tsochatzis@ucl.ac.uk

Received 23 January 2017; accepted 02 March 2017; published online 3 April 2017

DOI: https://doi.org/10.20524/aog.2017.0141 among HIV-positive individuals, even in the absence of hepatitis $\mathrm{C}(\mathrm{HCV})$ and $\mathrm{B}(\mathrm{HBV})$ co-infections [1,2]. Mild elevation of transaminases in HIV-positive individuals on ART is frequently reported [3], although the etiology is often indeterminate. In a study by Crum-Cianflone, elevated transaminases were found in 80 (27\%) of 299 HIV-monoinfected patients on ART and steatosis was detected on ultrasound (US) in $30 \%$, though in $51 \%$ the cause of liver dysfunction remained unexplained [4].

Fatty liver due to metabolic syndrome and/or ART exposure is an increasingly recognized cause of liver test abnormality in HIV-mono-infected patients [3]. Non-alcoholic fatty liver disease (NAFLD) is the hepatic manifestation of metabolic syndrome [5], but could also represent a long-term toxicity of ART as nucleoside reverse-transcriptase inhibitors (NRTIs) and to a lesser extent protease inhibitors (PIs), are associated with insulin resistance and mitochondrial toxicity [6]. Moreover, specific HIV-related factors, such as lipodystrophy or the HIV virus per se, may predispose to NAFLD $[7,8]$. NAFLD has a 
prevalence of $20 \%$ in the general population of industrialized countries, which rises to more than 50\% among those with HIV infection $[9,10]$.

Since NAFLD encompasses a wide spectrum of disease, ranging from simple steatosis to non-alcoholic steatohepatitis (NASH), fibrosis and eventually cirrhosis [11], the early detection of liver fibrosis prior to the onset of complications associated with decompensated liver disease is crucial. Noninvasive fibrosis tests are increasingly used for the assessment of fibrosis in patients with liver disease [12], including those who are HIV positive [13].

In this study, we retrospectively evaluated the prevalence and predictors of liver fibrosis in a cohort of HIV-monoinfected patients with persistently elevated transaminases, using simple serum noninvasive fibrosis panels, namely the FIB4 and APRI scores. In addition, we assessed the presence of hepatic steatosis and associated features in this cohort.

\section{Patients and methods}

\section{Study population}

This was a retrospective cross-sectional audit of consecutive HIV-mono-infected patients who attended the HIV dedicated outpatient service at Royal Free Hospital, London, UK, between January and December 2014. A clinical database was used to identify patients with persistently elevated transaminases in at least two measurements six months apart. Serum levels more than $39 \mathrm{IU} / \mathrm{L}$ and $41 \mathrm{IU} / \mathrm{L}$ were considered as abnormal for aspartate aminotransferase (AST) and alanine aminotransferase (ALT), respectively. Patients with evidence of HCV or HBV co-infection, as well as other documented causes of liver disease other than NAFLD were excluded. Further information for patients with persistently abnormal transaminases was retrieved from their clinical notes.

Laboratory investigations and epidemiological and clinical features were retrieved from the clinical database for all included patients. The presence of diabetes, hypertension and dyslipidemia was documented, as well as relevant medications.

HIV specific information was also obtained; this included time since diagnosis, duration of ART, most recent CD4+ count $\left(\mathrm{n} / \mathrm{mm}^{3}\right)$ and HIV viral load (RNA copies $\left./ \mathrm{mm}^{3}\right)$. HIV RNA copies $<40 / \mathrm{mL}$ defined virologic suppression.

\section{Liver fibrosis and steatosis assessment}

We obtained the results of abdominal US scans and transient elastography (TE) with FibroScan ${ }^{\circledast}$, which were performed as part of routine clinical care, to further investigate the liver test abnormalities and stage of fibrosis, respectively. US and TE findings were included if within 6 months of the date of transaminase measurement.

The presence of significant $(\geq \mathrm{F} 2$ METAVIR stage) or advanced ( $\geq$ F3 METAVIR stage) liver fibrosis was evaluated by
APRI and FIB4 scores, respectively. APRI consists of AST and platelet count in the formula:

APRI $=$ [AST levels $(\mathrm{IU} / \mathrm{L}) /$ AST upper limit of normal $(\mathrm{ULN})(\mathrm{IU} / \mathrm{L}) /$ Platelets $\left.\left(10^{9} / \mathrm{L}\right)\right] \times 100$

It has dual cutoffs for ruling out $(<0.5)$ or diagnosing $(>1.5)$ significant fibrosis [14]. FIB4 consists of age, AST, ALT and platelet count in the formula:

FIB4 $=[$ Age $($ years $) \times$ AST $(\mathrm{IU} / \mathrm{L})] /\left[\right.$ Platelets $\left(10^{9} / \mathrm{L}\right) \times$ $\operatorname{ALT}(\mathrm{IU} / \mathrm{L})]$

It also has dual cutoffs for ruling out $(<1.45)$ or diagnosing (>3.25) advanced fibrosis [15]. These tests usually have dual cutoffs: a high cutoff with high specificity and a low cutoff with high sensitivity. Depending on the clinical scenario and the disease prevalence, the low or high cutoff is used at the expense of increased false positives and false negatives respectively. If these cutoffs are combined, then the false positives and false negatives are minimized; however, a number of patients will fall in the indeterminate range of fibrosis (i.e., their score will be indeterminate, between the low and the high cutoff) and they will need either further noninvasive testing or a liver biopsy [16].

In a small subgroup of 19 patients, TE was performed with a FibroScan ${ }^{\circledast}$ system (Echosens; Paris, France), after at least 6 hours of fasting. Results in kilopascals $(\mathrm{kPa})$ were calculated as the mean of ten valid measurements, obtained by placing the probe on the patient's skin between the ribs at the level of the right lobe of the liver in a dorsal decubitus position. Only determinations of liver stiffness with an interquartile range for measurements within $30 \%$ and a ratio of success rate of measurements (number of total measurements/number of valid acquisition) $>60 \%$ were considered reliable. Liver stiffness values $>7.4 \mathrm{kPa}$ were considered suggestive of significant fibrosis.

Finally, 20 patients in the same cohort had previous liver biopsies as part of their routine clinical care. The presence and severity of steatosis, as well as the histological grading and staging were assessed according to the Brunt score [17].

\section{Statistical analysis}

All data were analyzed using the statistical package SPSS (version 22.0, IBM, New York, NY, USA). Statistical analysis was performed using $t$-test, ANOVA, Mann-Whitney test or Kruskal-Wallis test for comparisons of continuous variables between or among groups, the corrected chi-square method or two-tailed Fisher's exact test was used for comparisons of qualitative data, and Spearman's coefficient for correlations of quantitative data, when appropriate. Multivariate analysis was performed using logistic regression models to assess predictors of steatosis and fibrosis with APRI and FIB- 4 values of $>0.5$ and $>1.45$ respectively. Only variables with a P-value $\leq 0.10$ in the univariate analysis were entered in the multivariate analysis models. A two-tailed P-value $<0.05$ was considered to indicate a statistically significant difference between groups. 


\section{Results}

\section{Baseline characteristics}

Of 2398 consecutive patients with HIV mono-infection who were evaluated between January and December 2014, $156(6.5 \%)$ had persistently elevated transaminases in at least two measurements six months apart and were included in the analysis. The mean age of those included was 47.5 years and $91 \%$ were male. Clinical, biochemical and virological characteristics are reported in Table 1. In particular, 28 patients (17.9\%) had hypertension, 17 (10.9\%) had diabetes and 80 (51.9\%) had hyperlipidemia, while 47 (30.5\%) were on lipid-lowering medication.

AST and ALT values <2 times ULN were found in $93.6 \%$ and $81.4 \%$ of patients, respectively, 2-3 times ULN in at least one measurement in $3.8 \%$ and $14.1 \%$, and $>3$ times ULN in at least one measurement in $2.6 \%$ and $4.5 \%$ of the cohort.

Almost all patients were on ART $(n=154,97 \%)$, with a median length of treatment of 11 years (interquartile range [IQR] 0-26 years), and the majority of them had virological suppression $(\mathrm{n}=146,93.6 \%)$. Mean time since diagnosis was 14 years (IQR 2-30 years). CD4 counts $<200 / \mathrm{mm}^{3}$ were present only in $3(1.9 \%)$ patients and median viral load was 39 copies $/ \mathrm{mm}^{3}$ (IQR 39-2622).

Information about the specific treatment regimen was available for only 97 patients. The most common drugs used were NRTIs in $86(88.7 \%)$ of treated patients, while PIs and non-NRTIs were prescribed in $41(42.3 \%)$ and $46(47.4 \%)$ of patients, respectively.

\section{Prevalence and predictors of hepatic steatosis}

US examination was performed in $66(42.3 \%)$ patients and fatty liver was detected in the majority of these $(n=47,71.2 \%)$, as shown in Table 2. Nevertheless, no association was found between the presence of US steatosis and patients' clinical features, as shown in Table 3. In particular, there was no association between the presence of steatosis and metabolic features, namely hypertension, diabetes and dyslipidemia. Importantly, fatty liver was not significantly associated with the time since diagnosis, the length of ART, or the category of antiviral drugs used. On univariate analysis, fenofibrate use was significantly associated with the absence of steatosis on US $(\mathrm{P}=0.022)$, but this association was lost in the multivariate model.

\section{Prevalence and predictors of significant and advanced liver fibrosis}

Considering the high cutoff of $>1.5$ for APRI, we categorized $5(3.2 \%)$ patients as having significant fibrosis $(\geq \mathrm{F} 2)$, whereas values $<0.5$ excluded this diagnosis in $51.9 \%$ of them. On the other hand, FIB4 $>3.25$ suggested advanced fibrosis $(\geq \mathrm{F} 3)$ in $6(3.8 \%)$ patients, while values $\leq 1.45(<\mathrm{F} 3)$ were found in
Table 1 Characteristics of the cohort of HIV-mono-infected patients with deranged transaminases

\begin{tabular}{|c|c|}
\hline Characteristics & $\mathrm{N}=156$ \\
\hline Male sex (\%) & $143(91.7)$ \\
\hline Age, years & $47.5 \pm 8.5$ \\
\hline AST, IU/L & $41(22-299)$ \\
\hline ALT, IU/L & $56(29-372)$ \\
\hline GGT, U/L & $74(13-960)$ \\
\hline ALP, U/L & $86 \pm 34$ \\
\hline $\mathrm{PLT}, / \mathrm{mm}^{3}$ & $217 \pm 5$ \\
\hline Diabetes, n (\%) & $17(11)$ \\
\hline Hypertension, $\mathrm{n}(\%)$ & $28(18.2)$ \\
\hline Dyslipidemia, n (\%) & $81(51.9)$ \\
\hline Currently receiving statin, $\mathrm{n}(\%)$ & $47(30.5)$ \\
\hline Currently receiving fenofibrate, $\mathrm{n}(\%)$ & $7(4.5)$ \\
\hline Time since diagnosis, years & $14(2-30)$ \\
\hline Current treatment, n (\%) & $154(97)$ \\
\hline $\mathrm{PIs}^{*}, \mathrm{n}(\%)$ & $41(42.3)$ \\
\hline NRTIs*, n (\%) & $86(88.7)$ \\
\hline $\mathrm{NNRTs}^{*}, \mathrm{n}(\%)$ & $40(41.2)$ \\
\hline $\begin{array}{l}\text { Duration of ART years (amongst those } \\
\text { currently exposed) }\end{array}$ & $11(0-26)$ \\
\hline HIV-RNA, copies $/ \mathrm{mm}^{3}>100,000$ & 0 \\
\hline Virologic suppression & $146(93.6)$ \\
\hline $\mathrm{CD} 4+$, cells $/ \mathrm{mm}^{3}$ & $683(4-1900)$ \\
\hline
\end{tabular}

Data expressed as mean \pm SD for quantitative variables with normal distribution; data expressed as median (interquartile range) for quantitative variables without normal distribution, ${ }^{\star}$ Information on drug category available only in 97 patients $(62 \%)$

ALT, alanine aminotransferase; AST, aspartate aminotransferase; GGT, $\gamma$-glutamyltranspeptidase; ALP, alkaline phosphatase; PLT, platelets; PIs, protease inhibitors; NRTIs, nucleoside reverse transcriptase inhibitors; NNRTIs, non-nucleoside reverse transcriptase inhibitors

Table 2 Prevalence of liver disease in cohort of HIV-mono-infected patients with deranged transaminases

\begin{tabular}{lc}
\hline Variables & $\mathrm{N}=156$ \\
\hline Ultrasound examinations, $\mathrm{n}(\%)$ & $66(42.3)$ \\
Ultrasound steatosis, $\mathrm{n}(\%)$ & $47(71.2)$ \\
FIB4 score & \\
$1.45, \mathrm{n}(\%)$ & $105(67.3)$ \\
$1.46-3.24, \mathrm{n}(\%)$ & $45(28.8)$ \\
$\geq 3.25, \mathrm{n}(\%)$ & $6(3.8)$ \\
APRI index & \\
$0.5, \mathrm{n}(\%)$ & $81(51.9)$ \\
$0.51-1.49, \mathrm{n}(\%)$ & $70(44.9)$ \\
$\geq 1.5, \mathrm{n}(\%)$ & $5(3.2)$ \\
\hline
\end{tabular}


Table 3 Variables associated with the presence of US liver steatosis in the cohort of HIV-mono-infected patients

\begin{tabular}{|c|c|c|c|c|c|}
\hline \multirow[t]{2}{*}{ Patient characteristics } & \multirow[t]{2}{*}{ No steatosis $(\mathrm{N}=19)$} & \multirow[t]{2}{*}{ Steatosis $(\mathrm{N}=47)$} & \multirow{2}{*}{$\begin{array}{l}\text { Univariate analysis } \\
\text { P-value }\end{array}$} & \multicolumn{2}{|c|}{ Multivariate analysis } \\
\hline & & & & $\mathrm{P}$-value & OR $(95 \% \mathrm{CI})$ \\
\hline Age, years & $46.9 \pm 9.5$ & $47.9 \pm 8.5$ & 0.700 & & \\
\hline Male sex (\%) & $16(84.2)$ & $43(91.5)$ & 0.401 & & \\
\hline Diabetes mellitus, n (\%) & $0(0)$ & $6(12.8)$ & 0.171 & & \\
\hline Hypertension, n (\%) & $5(26.3)$ & $9(19.4)$ & 0.529 & & \\
\hline Dyslipidemia, n (\%) & $11(57.9)$ & $25(53.2)$ & 0.790 & & \\
\hline Statin, n (\%) & $7(36.8)$ & $15(31.9)$ & 0.779 & & \\
\hline Fenofibrate, n (\%) & $3(15.8)$ & $0(0)$ & 0.022 & NS & \\
\hline PLT, $\times 10^{3} / \mathrm{mm}^{3}$ & $218 \pm 51$ & $220 \pm 52$ & 0.836 & & \\
\hline AST, IU/L & $46 \pm 14$ & $46 \pm 22$ & 0.466 & & \\
\hline ALT, IU/L & $61 \pm 15$ & $66 \pm 28$ & 0.832 & & \\
\hline ALP, U/L & $92 \pm 37$ & $91 \pm 29$ & 0.835 & & \\
\hline GGT, U/L & $194 \pm 273$ & $97 \pm 86$ & 0.428 & & \\
\hline Duration since diagnosis, years & $15 \pm 8$ & $14 \pm 7$ & 0.451 & & \\
\hline Duration of treatment, years & $12 \pm 6$ & $16 \pm 6$ & 0.729 & & \\
\hline PIs, n (\%) & $4(21)$ & $13(27.7)$ & 1.000 & & \\
\hline NRTIs, n (\%) & $11(57.9)$ & $32(68.1)$ & 1.000 & & \\
\hline NNRTs, n (\%) & $6(31.6)$ & $14(29.8)$ & 0.737 & & \\
\hline Virologic suppression, n (\%) & $18(94.7)$ & $44(93.6)$ & 1.000 & & \\
\hline $\mathrm{CD} 4+<200 / \mathrm{mm}^{3}, \mathrm{n}(\%)$ & 0 & 0 & - & & \\
\hline FIB4 $>1.45, \mathrm{n}(\%)$ & $7(36.8)$ & $17(36.2)$ & 1.000 & & \\
\hline APRI >0.5, n (\%) & $10(52.6)$ & $25(53.2)$ & 1.000 & & \\
\hline
\end{tabular}

ALT, alanine aminotransferase; AST, aspartate aminotransferase; GGT, $\gamma$-glutamyltranspeptidase; ALP, alkaline phosphatase; PLT, platelets; PIs, protease inhibitors; NRTIs, nucleoside reverse transcriptase inhibitors; NNRTIs, non-nucleoside reverse transcriptase inhibitors; US, ultrasound; OR, odds ratio; 95\%CI, 95\% confidence interval

$67.3 \%$ of the cohort. Forty-five $(28.8 \%)$ and $70(44.9 \%)$ patients fell into the "indeterminate" diagnostic zone of FIB4 and APRI, respectively, as shown in Table 2.

For further analysis, and in keeping with the use of noninvasive tests for screening, we considered a threshold of 1.45 for FIB4 and of 0.5 of APRI to rule out the presence of advanced or significant fibrosis, respectively, in our cohort of HIV-mono-infected patients.

In univariate analysis, FIB4 $>1.45(\mathrm{n}=51,32.7 \%)$, was significantly associated with both time since HIV diagnosis ( $14 \pm 7$ vs. $16 \pm 6$ years, $P=0.019)$ and duration of ART ( $10 \pm 6$ vs. $13 \pm 6$ years, $\mathrm{P}=0.002)$. Moreover, diabetes was significantly more prevalent in patients with a FIB4 score $>1.45$ compared to those with values $<1.45$ ( $17.6 \%$ vs. $7.6 \%, \mathrm{P}=0.098)$. Nevertheless, in the multivariate analysis, only duration of ART maintained this association (OR 1.008, 95\%CI 1.000-1.016; $\mathrm{P}=0.04$ ) (Table 4).

On the other hand, APRI values $>0.5$ were found in 75 ( $48 \%)$ patients and only ALT levels were significantly increased in patients with evidence of significant fibrosis ( $78 \pm 49$ vs. $56 \pm 13$; OR 1.033 , 95\%CI $1.015-1.510, \mathrm{P}=0.01$ ). In contrast to FIB4, neither the time since HIV diagnosis nor the use of ART or the presence of diabetes was associated with an increased
APRI score. As with FIB4, no association with demographic or metabolic features was noted (Table 5). Interestingly, no relation was found between the presence of liver fibrosis assessed by both FIB4 and APRI scores and evidence of steatosis on US.

A small subset of patients also underwent TE ( $\mathrm{n}=19,12.1 \%)$ and a liver biopsy $(n=20,12.8 \%)$. Considering a threshold stiffness value of $7.4 \mathrm{kPa}, 4(21.1 \%)$ patients were classified as having significant fibrosis, while stiffness values more than 10 $\mathrm{kPa}$ were found in only 1 patient, suggesting more advanced liver disease.

Despite the small number of liver biopsies, histology showed NAFLD in 13 (65\%) patients, one of whom was also diagnosed with cirrhosis. Moreover, features of drug reaction were present in 2 cases, while 4 patients had non-specific histological changes and 1 biopsy was normal.

\section{Discussion}

In this study, we demonstrated that persistently abnormal transaminases are fairly prevalent in unselected HIV-mono- 
Table 4 Variables associated with the presence of liver fibrosis assessed by FIB4 in the whole cohort of HIV-mono-infected patients

\begin{tabular}{|c|c|c|c|c|c|}
\hline \multirow[t]{2}{*}{ Patient characteristics } & \multirow[t]{2}{*}{ FIB4 $\leq 1.45(\mathrm{~N}=105)$} & \multirow[t]{2}{*}{ FIB4 $>1.46(\mathrm{~N}=51)$} & \multirow{2}{*}{$\begin{array}{l}\text { Univariate analysis } \\
\text { P-value }\end{array}$} & \multicolumn{2}{|c|}{ Multivariate analysis } \\
\hline & & & & $\mathrm{P}$-value & OR $(95 \% \mathrm{CI})$ \\
\hline Age, years & - & - & - & & \\
\hline Male sex (\%) & $94(89.5)$ & $49(96.1)$ & 0.224 & & \\
\hline Diabetes mellitus, n (\%) & $8(7.6)$ & $9(17.6)$ & 0.098 & NS & \\
\hline Hypertension, n (\%) & $16(15.2)$ & $12(23.5)$ & 0.268 & & \\
\hline Dyslipidemia, n (\%) & $58(55.2)$ & $23(45.1)$ & 0.305 & & \\
\hline Statins, n (\%) & $32(30.5)$ & $15(29.4)$ & 1.000 & & \\
\hline Fenofibrate, n (\%) & $5(4.8)$ & $2(4)$ & 1.000 & & \\
\hline PLT, $\times 10^{3} / \mathrm{mm}^{3}$ & - & - & - & & \\
\hline ALT, IU/L & - & - & - & & \\
\hline AST, IU/L & - & - & - & & \\
\hline ALP, U/L & $88 \pm 33$ & $80 \pm 31$ & 0.138 & & \\
\hline GGT, U/L & $99 \pm 123$ & $156 \pm 191$ & 0.104 & & \\
\hline Time since HIV diagnosis, years & $14 \pm 7$ & $16 \pm 6$ & 0.019 & NS & \\
\hline Duration of ART, years & $10 \pm 6$ & $13 \pm 6$ & 0.002 & 0.041 & $1.008(1.000-1.016)$ \\
\hline PIs, n (\%) & $23(21.9)$ & $18(35.3)$ & 0.202 & & \\
\hline NRTIs, n (\%) & $55(52.4)$ & $31(60.8)$ & 1 & & \\
\hline NNRTs, n (\%) & $28(26.7)$ & $12(23.5)$ & 0.391 & & \\
\hline Virologic suppression, n (\%) & $98(9.3)$ & $48(94.1)$ & 1.000 & & \\
\hline $\mathrm{CD} 4+<200 / \mathrm{mm}^{3}, \mathrm{n}(\%)$ & $1(0.9)$ & $2(3.9)$ & 0.249 & & \\
\hline US steatosis, n (\%) & $30(28.6)$ & 17(33.3) & 1 & & \\
\hline
\end{tabular}

ALT, alanine aminotransferase; AST, aspartate aminotransferase; GGT, $\gamma$-glutamyltranspeptidase; ALP, alkaline phosphatase; PLT, platelets; PIs, protease inhibitors; NRTIs, nucleoside reverse transcriptase inhibitors; NNRTIs, non-nucleoside reverse transcriptase inhibitors; US, ultrasound; OR, odds ratio; $95 \%$ CI, 95\% confidence interval

infected patients $(156 / 2398,6.5 \%)$. More importantly, $4 \%$ of these patients had evidence of advanced fibrosis based on simple noninvasive fibrosis tests. Despite the presence of persistently deranged liver function tests (LFTs) in this group, there was inconsistent further investigation of these abnormalities; just $66(42 \%)$ patients had undergone a US examination and only $19(12 \%)$ had a TE, despite the noninvasiveness and relatively low cost of these techniques.

A rise in liver enzymes in HIV-mono-infected individuals on ART has been previously reported. A cross-sectional study by Sterling et al reported elevated transaminases in $20 \%$ of 679 patients and a positive association with high viral load and features of metabolic syndrome [18]. Maida et al studied a cohort of 3200 individuals with a long history of HIV infection and ART exposure and found abnormal LFTs in only $17(0.5 \%)$ patients, of whom $10(58.8 \%)$ had histologically advanced fibrosis (F3-F4) and clinically advanced liver disease. However, in this study harmful alcohol use and medications predisposing to steatosis were excluded [19]. On the other hand, in a prospective study of 2365 HIV-mono-infected individuals who were followed for a 5-year period, 385 (16\%) developed persistent ALT elevation, which was associated with increased body mass index, high HIV viral load and exposure to NRTIs, in particular zidovudine and stavudine [20].
The underlying etiology of increased LFTs in people who are HIV positive often remains unrecognized, as liver biopsy is not routinely performed. Nevertheless, many studies have indicated that NAFLD is more prevalent in this category of patients, potentially progressing to NASH and liver fibrosis.

A histological evaluation of a cohort of 20 HIV-monoinfected individuals with chronically increased LFTs demonstrated the presence of steatosis in 18 (60\%) patients, of whom 16 (88.9\%) had NASH and $13(72.2 \%)$ had fibrosis [21]. Morse et al biopsied a cohort of 62 patients with similar features and found steatosis in 45 (73\%), NASH in 34 (55\%) and bridging fibrosis in 11 (17\%) patients [22].

These data are similar to those of our study and indicate the necessity of screening HIV positive individuals for the presence of steatosis and fibrosis, especially when LFTs are deranged. In fact, in the subgroup of patients who underwent a liver US, NAFLD was present in the majority (47/66, 71.2\%). Moreover, even though a liver biopsy was performed in only in 20 patients, histology showed NAFLD in 13 (65\%), and NASH in $5(25 \%)$ patients, of whom one had cirrhosis.

The prevalence of steatosis is likely to be even higher than $70 \%$, as US can detect steatosis only when more than $20-30 \%$ of hepatocytes are involved. Importantly, in our study there was no significant association with metabolic, anthropometric 
Table 5 Variables associated with the presence of liver fibrosis assessed by APRI index in the whole cohort of HIV-mono-infected patients

\begin{tabular}{|c|c|c|c|c|c|}
\hline \multirow[t]{2}{*}{ Patient characteristics } & \multirow[t]{2}{*}{ APRI $\leq 0.5(\mathrm{~N}=81)$} & \multirow[t]{2}{*}{ APRI $>0.51 \quad(\mathrm{~N}=75)$} & \multirow{2}{*}{$\begin{array}{c}\text { Univariate analysis } \\
\text { P-value }\end{array}$} & \multicolumn{2}{|c|}{ Multivariate analysis } \\
\hline & & & & P-value & OR $(95 \% \mathrm{CI})$ \\
\hline Age, years & $47.1 \pm 8.3$ & $47.9 \pm 8.8$ & 0.57 & & \\
\hline Male sex (\%) & $73(89.5)$ & $70(96.1)$ & 0.568 & & \\
\hline Diabetes mellitus, n (\%) & $9(7.6)$ & $8(17.6)$ & 1 & & \\
\hline Hypertension, n (\%) & $14(15.2)$ & $14(23.5)$ & 0.836 & & \\
\hline Dyslipidemia, n (\%) & $45(55.2)$ & $36(45.1)$ & 0.423 & & \\
\hline PLT, $\times 10^{3} / \mathrm{mm}^{3}$ & - & - & - & & \\
\hline Statin, $\mathrm{n}(\%)$ & $23(30.5)$ & $24(29.4)$ & 0.601 & & \\
\hline Fenofibrate, n (\%) & $4(4.9)$ & $3(4)$ & 1.000 & & \\
\hline ALT, IU/L & $56 \pm 13$ & $78 \pm 49$ & 0.001 & 0.01 & $1.033(1.015-1.510)$ \\
\hline AST, IU/L & - & - & - & & \\
\hline ALP, U/L & $87 \pm 28$ & $85 \pm 37$ & 0.751 & & \\
\hline GGT, U/L & $85 \pm 64$ & $153 \pm 201$ & 0.223 & & \\
\hline Duration of infection, years & $15 \pm 7$ & $14 \pm 7$ & 0.425 & & \\
\hline Duration of treatment, years & $11 \pm 6$ & $11 \pm 6$ & 0.767 & & \\
\hline PIs, n (\%) & $19(23.4)$ & $22(29.3)$ & 0.311 & & \\
\hline NRTIs, n (\%) & $45(55.5)$ & $41(54.7)$ & 1.000 & & \\
\hline NNRTs, n (\%) & $20(24.7)$ & $20(26.7)$ & 0.685 & & \\
\hline $\mathrm{CD} 4+<200 / \mathrm{mm}^{3}, \mathrm{n}(\%)$ & $1(0.9)$ & $2(3.9)$ & 0.608 & & \\
\hline Virologic suppression, n (\%) & $76(93.8)$ & $70(93.3)$ & 1.000 & & \\
\hline US steatosis, n (\%) & $22(28.6)$ & $25(33.3)$ & 1.000 & & \\
\hline
\end{tabular}

ALT, alanine aminotransferase; AST, aspartate aminotransferase; GGT, $\gamma$-glutamyltranspeptidase; ALP, alkaline phosphatase; PLT, platelets; PIs, protease inhibitors; NRTIs, nucleoside reverse transcriptase inhibitors; NNRTIs, non-nucleoside reverse transcriptase inhibitors; US, ultrasound; OR, odds ratio; 95\%CI, 95\% confidence interval

and either infection- or ART-related features. However, as US reports were available for less than half of our cohort, this likely reflects under-powering of the study to detect any significant associations.

Conversely, two other studies reported a lower prevalence of NAFLD assessed by imaging techniques. Guaraldi et al detected steatosis by CT scan in $37 \%$ of 225 HIV-monoinfected patients, and found an association with cumulative NRTI exposure (OR 1.12, 95\%CI 1.03-1.22; $\mathrm{P}=<0.001$ ) and obesity (OR 1.07, 95\%CI 1.03-1.11; $\mathrm{P}<0.001$ ) [23]. In contrast, in another study, though steatosis was present on US in $31 \%$ and by histology in $33 \%$ of 216 HIV-mono-infected patients, there was no association with either duration of HIV infection or ART, as only obesity (OR 2.1, 95\%CI 1.6-2.8; $\mathrm{P}<0.001)$ and hypertriglyceridemia (OR $1.2,95 \%$ CI $1-1.5 ; \mathrm{P}=0.03$ ) reached statistical significance [24]. Nevertheless, in both studies less than $30 \%$ of patients had deranged LFTs; this could possibly explain the lower prevalence of steatosis compared to our findings.

Using the APRI and FIB4 scores, we classified 5 (3.2\%) and $6(3.8 \%)$ patients as potentially having significant and advanced fibrosis, respectively. Although TE appears to be the best noninvasive tool in HIV infected patients, showing excellent accuracy for the detection of liver cirrhosis and moderate accuracy for significant fibrosis $[25,26]$, it was available in only a small subset of the cohort, where it suggested significant fibrosis in 4 patients. On the other hand, our data suggest that $81(51.2 \%)$ and $105(67.3 \%)$ patients could be excluded from having significant and advanced fibrosis according to APRI and FIB4, respectively. Indeed, APRI and FIB4 have a higher diagnostic accuracy in ruling out rather than ruling in the presence of significant/advanced fibrosis and are ideal screening tests, as they have a negative predictive value of $>90 \%[15,27]$. Low FIB4 scores are associated with excellent liver-related outcomes in patients with NAFLD over a 10 -year period of follow up; therefore, these scores can be used in the HIV mono-infection setting [28].

The low prevalence of significant and advanced fibrosis based on simple noninvasive tests is compatible with previously published data. In a cohort of $818 \mathrm{HIV}$ positive women, the majority of patients $(86.6 \%)$ had FIB- 4 values $\leq 1.45$, while increasing FIB4 values were associated with higher HIV viral load [29]. Similarly, in a cohort of 225 HIV-mono-infected patients, 28 were classified as not having fibrosis on FIB-4 [23], while in another cohort of 62 patients FIB4 $\leq 1.45$ and $>3.25$ were found in 37 (59.7\%) and 5 (8\%) patients, respectively [22]. 
Conversely, a slightly higher prevalence of $8 \%$ of APRI values $>1.5$ was found in 432 HIV-mono-infected patients, and both diabetes and detectable HIV viremia were confirmed as risk factors for significant fibrosis [30]. However, the presence of obesity in $50 \%$ of the cohort could have contributed to the higher prevalence of significant fibrosis.

In our study, the duration of ART was the only factor independently associated with the presence of fibrosis based on FIB-4. The impact of ART on liver fibrosis is controversial, as highlighted by conflicting reports on worsening with long-term use of ART, especially didanosine or stavudine [9,31], absence of significant effect [22], or even improvement following commencement of PIs [32,33]. Furthermore, Mendeni et al followed a cohort of 1112 HIV-mono-infected patients for approximately 6 years and found that progression of fibrosis, assessed by APRI and FIB4, was prevented by viral control with early ART, provided that didanosine use was avoided [33].

Interestingly, in this study neither FIB4 nor APRI showed any association with metabolic features or increasing age. Diabetes was significantly associated with increased FIB4 values, but only in the univariate analysis. These data reflect the characteristics of our population, as the HIV patients were relatively young (mean age 47 years) and both diabetes and hypertension had a low prevalence, thus limiting the power of this study to detect any associations with fibrosis.

This study has limitations. First, it was a cross-sectional study, so it was not possible to define the dynamic process underlying the development of both steatosis and fibrosis in HIV positive patients. Therefore, these data should be assessed prospectively, in order to evaluate the true prognostic impact of hepatic steatosis and fibrosis in HIV. Second, the selection of patients on the basis of elevated transaminases could have led to an underestimation of fatty liver disease, given their lack of sensitivity in diagnosing NAFLD or NASH [34]. Third, detection of steatosis by US could also have underestimated the prevalence of NAFLD, as the accuracy of this technique is suboptimal. In addition, an element of undisclosed alcohol abuse could also have contributed to the high steatosis prevalence in this cohort.

In conclusion, the presence of liver disease is underinvestigated in HIV-mono-infected patients, despite deranged LFTs. Such abnormalities are often associated with the presence of NAFLD, and could potentially evolve into progressive liver fibrosis. A potential role for ART in this scenario has yet to be determined, but it is likely that NAFLD could represent a long-term toxicity of antiviral therapy, as suggested by the association of the length of antiviral treatment and liver fibrosis and evidence from other studies. A wide range of noninvasive tools for the assessment of liver fibrosis are currently available; therefore, noninvasive screening for the presence of NAFLD and fibrosis should be a part of the routine clinical management of HIV-mono-infected patients receiving antiretroviral treatment. Moreover, aggressive treatment of the metabolic syndrome components should be undertaken in HIV positive patients with steatosis, given the well-established association of NAFLD with increased cardiovascular morbidity and mortality [35].

\section{Summary Box}

\section{What is already known:}

- Liver disease is an important cause of morbidity and mortality among HIV-infected patients, even in the absence of hepatitis $\mathrm{C}(\mathrm{HCV})$ and $\mathrm{B}(\mathrm{HBV})$ co-infections

- Abnormal liver tests in HIV mono-infection are frequently reported and nonalcoholic fatty liver disease (NAFLD) is increasingly recognized as potential cause

- Noninvasive fibrosis tests are widely used for the assessment of fibrosis in patients with liver disease, including HIV-positive individuals

\section{What the new findings are:}

- Despite the high prevalence of liver test abnormalities in HIV patients, liver disease is frequently under-investigated

- The duration of antiretroviral therapy seems to have an impact on the onset of hepatic steatosis and its progression to fibrosis. Therefore, NAFLD could represent a long-term toxicity of this pharmacological treatment

- Simple noninvasive tests such as APRI and FIB4 can be used for the triaging of patients for dedicated hepatological follow up and/or liver biopsy

\section{References}

1. Palella FJ Jr, Delaney KM, Moorman AC, et al. Declining morbidity and mortality among patients with advanced human immunodeficiency virus infection. HIV Outpatient Study Investigators. N Engl J Med 1998;338:853-860.

2. Bica I, McGovern B, Dhar R, et al. Increasing mortality due to endstage liver disease in patients with human immunodeficiency virus infection. Clin Infect Dis 2001;32:492-497.

3. Pol S, Lebray P, Vallet-Pichard A. HIV infection and hepatic enzyme abnormalities: intricacies of the pathogenic mechanisms. Clin Infect Dis 2004;38 Suppl 2:S65-S72.

4. Crum-Cianflone N, Collins G, Medina S, et al. Prevalence and factors associated with liver test abnormalities among human immunodeficiency virus-infected persons. Clin Gastroenterol Hepatol 2010;8:183-191.

5. Tsochatzis EA, Manolakopoulos S, Papatheodoridis GV, Archimandritis AJ. Insulin resistance and metabolic syndrome in chronic liver diseases: old entities with new implications. Scand J Gastroenterol 2009;44:6-14.

6. Núñez M. Hepatotoxicity of antiretrovirals: incidence, mechanisms and management. J Hepatol 2006;44:S132-S139.

7. McGovern BH, Ditelberg JS, Taylor LE, et al. Hepatic steatosis is associated with fibrosis, nucleoside analogue use, and hepatitis $\mathrm{C}$ 
virus genotype 3 infection in HIV-seropositive patients. Clin Infect Dis 2006;43:365-372.

8. Leow MK, Addy CL, Mantzoros CS. Clinical review 159: Human immunodeficiency virus/highly active antiretroviral therapy-associated metabolic syndrome: clinical presentation, pathophysiology, and therapeutic strategies. J Clin Endocrinol Metab 2003;88:1961-1976.

9. Blanco F, Barreiro P, Ryan P, et al. Risk factors for advanced liver fibrosis in HIV-infected individuals: role of antiretroviral drugs and insulin resistance. J Viral Hepat 2011;18:11-16.

10. Lombardi R, Sambatakou H, Mariolis I, Cokkinos D, Papatheodoridis GV, Tsochatzis EA. Prevalence and predictors of liver steatosis and fibrosis in unselected patients with HIV monoinfection. Dig Liver Dis 2016;48:1471-1477.

11. Vuppalanchi R, Chalasani N. Nonalcoholic fatty liver disease and nonalcoholic steatohepatitis: Selected practical issues in their evaluation and management. Hepatology 2009;49:306-317.

12. Buzzetti E, Lombardi R, De Luca L, Tsochatzis EA. Noninvasive assessment of fibrosis in patients with nonalcoholic fatty liver disease. Int J Endocrinol 2015;2015:343828.

13. Tsochatzis EA, Castera L. Assessing liver disease in HIV-HCV coinfected patients. Curr Opin HIV AIDS 2015;10:316-322.

14. Wai CT, Greenson JK, Fontana RJ, et al. A simple noninvasive index can predict both significant fibrosis and cirrhosis in patients with chronic hepatitis C. Hepatology 2003;38:518-526.

15. Sterling RK, Lissen E, Clumeck N, et al; APRICOT Clinical Investigators. Development of a simple noninvasive index to predict significant fibrosis in patients with HIV/HCV coinfection. Hepatology 2006;43:1317-1325.

16. Crossan C, Tsochatzis EA, Longworth L, et al. Cost-effectiveness of non-invasive methods for assessment and monitoring of liver fibrosis and cirrhosis in patients with chronic liver disease: systematic review and economic evaluation. Health Technol Assess 2015;19:1-409, v-vi.

17. Brunt EM, Janney CG, Di Bisceglie AM, NeuschwanderTetri BA, Bacon BR. Nonalcoholic steatohepatitis: a proposal for grading and staging the histological lesions. Am J Gastroenterol 1999;94:2467-2474.

18. Sterling RK, Chiu S, Snider K, Nixon D. The prevalence and risk factors for abnormal liver enzymes in HIV-positive patients without hepatitis B or C coinfections. Dig Dis Sci 2008;53:1375-1382.

19. Maida I, Núñez M, Ríos MJ, et al. Severe liver disease associated with prolonged exposure to antiretroviral drugs. J Acquir Immune Defic Syndr 2006;42:177-182.

20. Kovari H, Ledergerber B, Battegay $M$, et al. Incidence and risk factors for chronic elevation of alanine aminotransferase levels in HIV-infected persons without hepatitis B or C virus co-infection. Clin Infect Dis 2010;50:502-511.

21. Ingiliz P, Valantin MA, Duvivier C, et al. Liver damage underlying unexplained transaminase elevation in human immunodeficiency virus-1 mono-infected patients on antiretroviral therapy.
Hepatology 2009;49:436-442.

22. Morse CG, McLaughlin M, Matthews L, et al. Nonalcoholic steatohepatitis and hepatic fibrosis in HIV-1-monoinfected adults with elevated aminotransferase levels on antiretroviral therapy. Clin Infect Dis 2015;60:1569-1578.

23. Guaraldi G, Squillace N, Stentarelli C, et al. Nonalcoholic fatty liver disease in HIV-infected patients referred to a metabolic clinic: prevalence, characteristics, and predictors. Clin Infect Dis 2008;47:250-257.

24. Crum-Cianflone N, Dilay A, Collins G, et al. Nonalcoholic fatty liver disease among HIV-infected persons. J Acquir Immune Defic Syndr 2009;50:464-473.

25. de Lédinghen V, Douvin C, Kettaneh A, et al. Diagnosis of hepatic fibrosis and cirrhosis by transient elastography in HIV/ hepatitis C virus-coinfected patients. J Acquir Immune Defic Syndr 2006;41:175-179.

26. Vergara S, Macías J, Rivero A, et al; Grupo para el Estudio de las Hepatitis Viricas de la SAEI. The use of transient elastometry for assessing liver fibrosis in patients with HIV and hepatitis $C$ virus coinfection. Clin Infect Dis 2007;45:969-974.

27. Vallet-Pichard A, Mallet V, Nalpas B, et al. FIB-4: an inexpensive and accurate marker of fibrosis in HCV infection. Comparison with liver biopsy and fibrotest. Hepatology 2007;46:32-36.

28. Angulo P, Bugianesi E, Bjornsson ES, et al. Simple noninvasive systems predict long-term outcomes of patients with nonalcoholic fatty liver disease. Gastroenterology 2013;145:782-789.e4.

29. Blackard JT, Welge JA, Taylor LE, et al. HIV mono-infection is associated with FIB-4 - A noninvasive index of liver fibrosis - in women. Clin Infect Dis 2011;52:674-680.

30. DallaPiazza M, Amorosa VK, Localio R, Kostman JR, Lo Re V $3^{\text {rd }}$. Prevalence and risk factors for significant liver fibrosis among HIV-monoinfected patients. BMC Infect Dis 2010;10:116.

31. Al-Mohri H, Murphy T, Lu Y, Lalonde RG, Klein MB. Evaluating liver fibrosis progression and the impact of antiretroviral therapy in HIV and hepatitis C coinfection using a noninvasive marker. J Acquir Immune Defic Syndr 2007;44:463-469.

32. Han SH, Kim SU, Kim CO, et al. Abnormal liver stiffness assessed using transient elastography (Fibroscan ${ }^{\circ}$ ) in HIV-infected patients without $\mathrm{HBV} / \mathrm{HCV}$ coinfection receiving combined antiretroviral treatment. PLoS One 2013;8:e52720.

33. Mendeni M, Focà E, Gotti D, et al. Evaluation of liver fibrosis: concordance analysis between noninvasive scores (APRI and FIB-4) evolution and predictors in a cohort of HIV-infected patients without hepatitis $\mathrm{C}$ and $\mathrm{B}$ infection. Clin Infect Dis 2011;52:1164-1173.

34. Mofrad P, Contos MJ, Haque M, et al. Clinical and histologic spectrum of nonalcoholic fatty liver disease associated with normal ALT values. Hepatology 2003;37:1286-1292.

35. Adams LA, Lymp JF, St Sauver J, et al. The natural history of nonalcoholic fatty liver disease: a population-based cohort study. Gastroenterology 2005;129:113-121. 